\title{
Comparing Error Minimized Extreme Learning Machines and Support Vector Sequential Feed-forward Neural Networks *
}

\author{
Enrique Romero and René Alquézar
}

June 2, 2010

\begin{abstract}
Recently, error minimized extreme learning machines (EM-ELMs) have been proposed as a simple and efficient approach to build single-hidden-layer feedforward networks (SLFNs) sequentially. They add random hidden nodes one by one (or group by group) and update the output weights incrementally to minimize the sum-of-squares error in the training set. Other very similar methods that also construct SLFNs sequentially had been reported earlier with the main difference that their hidden-layer weights are a subset of the data instead of being random. By analogy with the concept of support vectors original of support vector machines (SVMs), these approaches can be referred to as support vector sequential feed-forward neural networks (SV-SFNNs), and they are a particular case of the Sequential Approximation with Optimal Coefficients and Interacting Frequencies (SAOCIF) method. In this paper, it is firstly shown that EM-ELMs can also be cast as a particular case of SAOCIF. In particular, EM-ELMs can easily be extended to test some number of random candidates at each step and select the best of them, as SAOCIF does. Moreover, it is demonstrated that the cost of the calculation of the optimal output-layer weights in the originally proposed EM-ELMs can be improved if it is replaced by the one included in SAOCIF. Secondly, we present the results of an experimental study on 10 benchmark classification and 10 benchmark regression data sets, comparing EM-ELMs and SV-SFNNs, that was carried out under the same conditions for the two models. Although both models have the same (efficient) computational cost, a statistically significant improvement in generalization performance of SV-SFNNs $v s$. EM-ELMs was found in 12 out of the 20 benchmark problems.
\end{abstract}

${ }^{*}$ This work was supported in part by the Ministerio de Ciencia e Innovación (MICINN), under project TIN2009-13895-C02-01. 


\section{Introduction}

Feed-forward Neural Networks (FNNs) are a popular machine learning approach for classification and regression problems with very interesting properties (see, for example, [2]). As a specific type of FNNs, the single-hidden-layer feed-forward networks (SLFNs) play an important role in practical applications. Since the optimal number of hidden nodes is problem dependent and unknown in advance, users usually choose the number of hidden nodes by trial-and-error. Once the architecture is fixed, an iterative learning algorithm such as back-propagation gradient descent is usually applied to adjust the weights in the output and hidden layers simultaneously.

There exist, however, FNN models that construct the network sequentially, so that the number of hidden units is a result of the learning process rather than being fixed a priori. For a review of constructive FNNs see, for example, [8]. Recently, error minimized extreme learning machines (EM-ELMs) have been proposed as a simple and efficient approach to build SLFNs sequentially [4]. EM-ELMs are an incremental extension of the previously presented extreme learning machines (ELMs) [6]. Both methods use random hidden nodes and find the output weights to minimize the sumof-squares error in the training set by solving a linear system of equations. The specific features of EM-ELMs with respect to ELMs are that they add random hidden nodes one by one (or group by group) and update the output weights incrementally in an efficient way by taking advantage of the incremental construction of the hidden-layer output matrix involved in the linear system. Other recent extensions of ELMs can be found in $[17,9]$.

Very similar methods that also construct SLFNs sequentially had been reported earlier ([3], [16], [12]). They all find the optimal linear weights of the output layer by solving the same linear system. In fact, the idea of adding random hidden units was already stated in the Sequential Approximation with Optimal Coefficients and Interacting Frequencies (SAOCIF) algorithm ([11], [12]) as a possible strategy to be used and, as shown in [12], the solution of the linear system can be computed efficiently thanks to the incremental construction of the hidden-layer output matrix. The EM-ELMs and SAOCIF with random selection strategy can easily be shown to be essentially equivalent (see Section 2), although it will be demonstrated in Section 3.1 that the cost of the output-layer weight computation described in [4] is greater than the cost of the corresponding one in SAOCIF described in [12].

Another strategy proposed in [11], [12] to be used within SAOCIF was to take hidden-layer weights always as a subset of the data (input strategy). In this case, the resulting method is equivalent to the Orthogonal Least Squares Learning algorithm [3] and to Kernel Matching Pursuit with pre-fitting [16]. All of them select the hiddenlayer weights among the input vectors. By analogy with the concept of support vectors original of support vector machines (SVMs) [15], these approaches can be referred to as support vector sequential feed-forward neural networks (SV-SFNNs) [13].

SV-SFNNs and SVMs were compared experimentally in [13]. Very similar accuracies were found, although computational times were lower for SVMs. Regarding the number of support vectors, SV-SFNNs constructed models with less hidden units than standard SVMs and in the same range as "sparse" SVMs [7]. On the other hand, EM-ELMs were compared in [4] with other sequential algorithms, namely resource al- 
location network (RAN) [10] and minimum resource allocation network (MRAN) [18], as well as with the original ELMs [6]. EM-ELMs obtained better performance and less training time than RAN and MRAN, and a similar performance but less training time than ELMs.

This work focuses on the comparison of EM-ELMs (i.e. SAOCIF with random strategy) and SV-SFNNs (i.e. SAOCIF with input strategy). An experimental study on 10 benchmark data sets for classification problems and 10 benchmark data sets for regression problems is presented in which the two methods are compared in the same conditions and using the same software. Since both approaches can be adjusted to have the same computational cost (each candidate weight vector for a hidden unit is either generated randomly or selected randomly among the input vectors), the goal is finding out whether there is any difference in generalization performance between EM-ELMs and SV-SFNNs. In other words, does the use of inputs (support vectors) as hidden unit weights provide any advantage over pure random selection?

Although it may be argued that the input strategy is using some sort of information present in the training data (to set the hidden-layer weights) whereas the random strategy is not, this does not necessarily imply that the generalization performance of the former has to be better than that of the latter. Therefore, an empirical comparative study on a wide range of problems is interesting to assess this point. Moreover, it should be noted that, on one hand, EM-ELMs have been proposed to build SLFNs as an alternative superior (due to their simplicity, efficiency and effectiveness) to common neural net approaches like backpropagation gradient descent [4] and, on the other hand, input vectors are rarely used as hidden-layer weights in SLFNs, especially in the case of additive units (i.e. two-layer perceptrons).

\section{Background}

The output function of an SLFN (i.e. a fully connected FNN with a single hidden layer of $N_{h}$ units and $m$ linear output units) can be expressed as a linear combination of simple (basis) functions:

$$
f_{N_{h}}(\mathbf{x})=\lambda_{0}+\sum_{i=1}^{N_{h}} \lambda_{i} \varphi\left(\boldsymbol{\omega}_{i}, b_{i}, \mathbf{x}\right)
$$

where $\boldsymbol{\omega}_{i} \in \mathbb{R}^{n}$ and $b_{i} \in \mathbb{R}$ are the learning parameters of the hidden units, $\lambda_{i} \in \mathbb{R}^{m}$ are the output-layer weights connecting the $i$-th hidden unit to the $m$ output units, $\varphi$ is the activation function of the hidden units, $\varphi\left(\boldsymbol{\omega}_{i}, b_{i}, \mathbf{x}\right)$ is the output of the $i$-th hidden unit with respect to the input $\mathbf{x}$, and $\lambda_{0} \in \mathbb{R}^{m}$ denotes the bias terms (if any) of the linear output units.

Although a lot of activation functions $\varphi$ (even not neuron alike) can be used that allow universal approximation capability, the more usual choices are the Gaussian RBF applied to a distance between an input vector and a centre

$$
\varphi\left(\boldsymbol{\omega}_{i}, b_{i}, \mathbf{x}\right)=\operatorname{gau}\left(b_{i}\left\|\mathbf{x}-\boldsymbol{\omega}_{i}\right\|\right)
$$


and the sigmoid (e.g. hyperbolic tangent) applied to a scalar product of the input and weight vectors (this will be referred to as a sigmoid additive unit [4])

$$
\varphi\left(\boldsymbol{\omega}_{i}, b_{i}, \mathbf{x}\right)=\operatorname{tnh}\left(\boldsymbol{\omega}_{i} \cdot \mathbf{x}+b_{i}\right) .
$$

For Gaussian RBF units, $\boldsymbol{\omega}_{i} \in \mathbb{R}^{n}$ and $b_{i} \in \mathbb{R}^{+}$are the centre and the impact factor of the $i$-th RBF unit. For sigmoid additive units, $\boldsymbol{\omega}_{i} \in \mathbb{R}^{n}$ is the weight vector connecting the input layer to the $i$-th hidden unit and $b_{i} \in \mathbb{R}$ is the bias of the $i$-th hidden unit. In our experiments presented in Section 3, a third activation function has also been tested, the sin applied to the scalar product (i.e. a sin additive unit)

$$
\varphi\left(\boldsymbol{\omega}_{i}, b_{i}, \mathbf{x}\right)=\sin \left(\boldsymbol{\omega}_{i} \cdot \mathbf{x}+b_{i}\right) .
$$

For a given set of training examples $\left\{\left(\mathbf{x}_{j}, \mathbf{t}_{j}\right)\right\}_{j=1}^{L} \subset \mathbb{R}^{n} \times \mathbb{R}^{m}$, if the outputs of the network are equal to the targets, we have

$$
f_{N_{h}}\left(\mathbf{x}_{j}\right)=\sum_{i=1}^{N_{h}} \lambda_{i} \varphi\left(\boldsymbol{\omega}_{i}, b_{i}, \mathbf{x}_{j}\right)=\mathbf{t}_{j}, \quad j=1, \ldots, L .
$$

Equation (5) can be written compactly as

$$
\mathbf{H} \boldsymbol{\lambda}=\mathbf{T}
$$

where $\mathbf{H}$ is an $L \times N_{h}$ matrix called the hidden-layer output matrix of the network $\left(\mathbf{H}_{j i}=\varphi\left(\boldsymbol{\omega}_{i}, b_{i}, \mathbf{x}_{j}\right)\right), \boldsymbol{\lambda}$ is an $N_{h} \times m$ matrix containing the output-layer weights, and $\mathbf{T}$ is an $L \times m$ matrix containing the target values in the training set. The output layer biases can be added by including in $\mathrm{H}$ a first column with a fixed value of 1 (and increasing $N_{h}$ by 1 ).

Normally, the number of training examples $L$ will be much greater than the number of hidden units $N_{h}$ and an exact solution of (6) cannot be expected. Then, the usual cost function in SLFNs (and in general in FNNs) is the sum-of-squares error

$$
E=\frac{1}{2} \sum_{j=1}^{L}\left\|f_{N_{h}}\left(\mathbf{x}_{j}\right)-\mathbf{t}_{j}\right\|^{2} .
$$

It is well known (e.g. [2]) that, to minimize $E$, the optimal output-layer weights can be computed as

$$
\hat{\boldsymbol{\lambda}}=\mathbf{H}^{\dagger} \mathbf{T} \quad \text { where } \quad \mathbf{H}^{\dagger}=\left(\mathbf{H}^{T} \mathbf{H}\right)^{-1} \mathbf{H}^{T}
$$

is the pseudo-inverse (or Moore-Penrose generalized inverse) of the hidden-layer output matrix $\mathbf{H}$. The sum-of-squares error can be expressed as

$$
E(\mathbf{H})=\frac{1}{2}\|\mathbf{H} \boldsymbol{\lambda}-\mathbf{T}\|^{2}=\frac{1}{2}\left\|\mathbf{H H}^{\dagger} \mathbf{T}-\mathbf{T}\right\|^{2} .
$$




\subsection{Error Minimized Extreme Learning Machines (EM-ELMs)}

Huang et al. [5] have shown that SLFNs with random weights in the hidden layer have universal approximation capability for many different choices of the activation function, including the ones stated in eqs. 2 to 4 . Based on this result, they propose the ELMs learning algorithm [6], which can be summarized as follows:

Algorithm for ELMs: Given a set of training examples $\left\{\left(\mathbf{x}_{j}, \mathbf{t}_{j}\right)\right\}_{j=1}^{L} \subset \mathbb{R}^{n} \times \mathbb{R}^{m}$, the hidden-layer activation function $\varphi(\boldsymbol{\omega}, b, \mathbf{x})$, and an a-priori fixed number $N_{h}$ of hidden units:

1) randomly assign hidden-unit parameters $\left(\boldsymbol{\omega}_{i}, b_{i}\right), i=1, \ldots, N_{h}$

2) calculate the hidden-layer output matrix $\mathbf{H}$

3) calculate the output-layer weight matrix $\lambda$ using (8)

In order to avoid the need of setting in advance the number $N_{h}$ of hidden units and to reduce the training computational time, a fast sequential extension of the ELMs algorithm called EM-ELMs has been recently reported by Feng et al. [4].

Algorithm for EM-ELMs: Given a set of training examples $\left\{\left(\mathbf{x}_{j}, \mathbf{t}_{j}\right)\right\}_{j=1}^{L} \subset \mathbb{R}^{n} \times$ $\mathbb{R}^{m}$, the maximum number of hidden units $N_{\max }$, and the expected learning accuracy $\epsilon$ :

1) Initialization phase:

- initialize the SLFN with a small group of $N_{0}$ randomly generated hidden units

$\left(\boldsymbol{\omega}_{i}, b_{i}\right), i=1, \ldots, N_{0}$

- calculate the hidden-layer output matrix $\mathbf{H}_{0}$

- calculate the corresponding output error

2) Recursively growing phase:

- let $k:=0$

- while $N_{k}<N_{\max }$ and $E\left(\mathbf{H}_{k}\right)>\epsilon$ do

- let $k:=k+1$

- randomly add $\delta N_{k}$ hidden units to the existing SLFN; the total number of hidden units becomes $N_{k}=N_{k-1}+\delta N_{k}$ and the corresponding hidden-layer output matrix $\mathbf{H}_{k}=\left[\mathbf{H}_{k-1}, \boldsymbol{\delta} \mathbf{H}_{k}\right]$, where $\delta \mathbf{H}_{k}$ contains the new $\delta N_{k}$ columns computed - the output-layer weights $\boldsymbol{\lambda}$ are updated as

- end while

$$
\begin{aligned}
\boldsymbol{\lambda}_{k}=\mathbf{H}_{k}^{\dagger} \mathbf{T} & =\left[\begin{array}{c}
\mathbf{U}_{k} \\
\mathbf{D}_{k}
\end{array}\right] \mathbf{T}, \text { where } \\
\mathbf{D}_{k} & =\left(\left(\mathbf{I}-\mathbf{H}_{k-1} \mathbf{H}_{k-1}^{\dagger}\right) \boldsymbol{\delta} \mathbf{H}_{k}\right)^{\dagger} \text { and } \\
\mathbf{U}_{k} & =\mathbf{H}_{k-1}^{\dagger}\left(\mathbf{I}-\boldsymbol{\delta} \mathbf{H}_{k} \mathbf{D}_{k}\right)
\end{aligned}
$$

\subsection{Sequential Approximation with Optimal Coefficients and In- teracting Frequencies (SAOCIF)}

A rather general constructive method for SLFNs, called SAOCIF, was proposed in [11], [12] by Romero and Alquézar. The specific features of SAOCIF are: $i$ ) the optimal (in a least squares sense) output-layer weights are recalculated each time a hidden unit is 
added by solving a linear equations system, and ii) the added hidden unit is selected among a set of candidates taking into account its interaction with the previously added hidden units (i.e. to minimize together the training error). The SAOCIF algorithm can be described as follows:

Algorithm for SAOCIF: Given a set of training examples $\left\{\left(\mathbf{x}_{j}, \mathbf{t}_{j}\right)\right\}_{j=1}^{L} \subset \mathbb{R}^{n} \times \mathbb{R}^{m}$, the maximum number of hidden units $N_{\max }$, a strategy to generate the candidates, the maximum number of candidates for any hidden unit $C_{\max }$, and the expected learning accuracy $\epsilon$ :

- let $N:=0 / /$ in this case, $N$ is equivalent to both $k$ and $N_{k}$ in EM-ELMs, because $\delta N_{k}=1$ for SAOCIF

- let $\mathbf{H}_{0}=[]$

- repeat

- let $N:=N+1$

- let $c:=0 / / \mathrm{c}$ is the number of valid candidates tested for the $N^{\text {th }}$ hidden unit

- while $c<C_{\max }$ do

- generate a candidate $(\boldsymbol{\omega}, b)$ for the $N^{\text {th }}$ hidden unit with the given strategy, and store in a temporary matrix $\mathbf{H}$ the corresponding hidden-layer output matrix $\mathbf{H}=\left[\mathbf{H}_{N-1}, \boldsymbol{\delta} \mathbf{H}\right]$, where $\boldsymbol{\delta} \mathbf{H}$ contains the new column computed

- find the optimal output-layer weights $\boldsymbol{\lambda}=\mathbf{H}^{\dagger} \mathbf{T}$ for the current candidate $(\boldsymbol{\omega}, b)$ using the incremental method described in [12] (equivalent in essence to the incremental method described in the algorithm for EM-ELMs, see Section 3.1)

- if the current candidate is considered valid (linear system without numerical problems, etc) then

- let $c:=c+1$

- calculate the corresponding output error

$$
E(\mathbf{H})=\frac{1}{2}\left\|\mathbf{H H}^{\dagger} \mathbf{T}-\mathbf{T}\right\|^{2}
$$

- if $E(\mathbf{H})$ is the minimum error found for the tested candidates in the current loop then

$$
\text { - let }\left(\boldsymbol{\omega}_{N}, b_{N}\right)=(\boldsymbol{\omega}, b) ; \quad \boldsymbol{\lambda}_{N}=\boldsymbol{\lambda} ; \quad \mathbf{H}_{N}=\mathbf{H}
$$

- end if

- end if

- end while

- until $N=N_{\max }$ or $E\left(\mathbf{H}_{N}\right) \leqslant \epsilon$

Note that if we set $C_{\max }=1$ and a random strategy to generate the candidates in the SAOCIF algorithm and we set $\delta N_{k}=1$ (for all $k$ ) in the algorithm for EM-ELMs, then both algorithms are essentially equivalent.

\subsection{Support Vector Sequential Feed-forward Neural Networks (SV- SFNNs)}

Apart from the random strategy, other possibilities are allowed in the SAOCIF approach to generate the candidates. In particular, let us define the input strategy as the 
one in which the candidates are only selected among the input examples in the training set; more precisely, $\boldsymbol{\omega}=\mathbf{x}_{j}$, for some $j$ not already used, and $b$ is a constant depending on the activation function (e.g. $b=1$ for RBF units and $b=0$ for additive units). Then, if we set $C_{\max }=L-N+1$ and generate the candidates using the input strategy, then the resulting method, which has been called SV-SFNNs [13], is equivalent to the Orthogonal Least Squares Learning (OLSL) algorithm [3] and to Kernel Matching Pursuit with pre-fitting (KMP-prefit) [16]. Actually, OLSL was only proposed for RBF units and KMP-prefit for kernel-based activation functions, while SAOCIF with input strategy permits as well any other activation function with universal approximation capabilities (e.g. sinusoidal additive units).

\section{Comparing EM-ELMs and SV-SFNNs}

This section compares EM-ELMs with SV-SFNNs and explains the methodology followed in the experiments.

\subsection{Analysis of the Computational Cost}

The computational complexity of the algorithm for EM-ELMs is dominated by the computation of $\mathbf{D}_{k}$ and $\mathbf{U}_{k}$, which involves, as intermediate steps, the computation of $L \times L$ matrices, that is expensive in terms of time and memory. Since $L$ will usually be much larger than $N_{\max }$, and assuming that $N_{k-1}>\delta N_{k}$, the computational cost to obtain $\mathbf{D}_{k}$ and $\mathbf{U}_{k}$ is $O\left(L^{2} \cdot N_{k-1}\right)$. However, this computation can be done with lower computational cost, as explained next.

Defining $\mathbf{A}=\mathbf{H}_{k-1}^{T} \mathbf{H}_{k-1}, \mathbf{v}=\mathbf{H}_{k-1}^{T} \delta \mathbf{H}_{k}, \mathbf{u}=\delta \mathbf{H}_{k}^{T} \boldsymbol{\delta} \mathbf{H}_{k}, \boldsymbol{\alpha}=\mathbf{H}_{k-1}^{T} \mathbf{T}$ and $\boldsymbol{\beta}=\boldsymbol{\delta} \mathbf{H}_{k}^{T} \mathbf{T}$, it is very easy to verify that

$$
\begin{aligned}
& \mathbf{D}_{k} \mathbf{T}=\left(\mathbf{u}-\mathbf{v}^{T} \mathbf{A}^{-1} \mathbf{v}\right)^{-1}\left(\boldsymbol{\beta}-\mathbf{v}^{T} \mathbf{A}^{-1} \boldsymbol{\alpha}\right)=\boldsymbol{\eta} \\
& \mathbf{U}_{k} \mathbf{T}=\mathbf{A}^{-1}(\boldsymbol{\alpha}-\mathbf{v} \boldsymbol{\eta})
\end{aligned}
$$

that is a generalization of the incremental method described in [12] for $m$ outputs and adding $\delta N_{k}$ hidden units in the same step. The computation of (10) and (11) is faster than those of the original algorithm for EM-ELMs because its computational cost is $O\left(L \cdot N_{k-1}^{2}\right)$.

Therefore, although the computational cost of the original algorithms EM-ELMs and SV-SFNNs is not the same, they can be easily made equivalent.

For $C_{\max }>1$, $\mathbf{A}$ can be computed for the first candidate, kept in memory and recovered for the rest of candidates. Therefore, the computational cost for $C_{\max }>1$ is lower than $C_{\max }$ times the computational cost for $C_{\max }=1$. More precisely, with this optimization the computational cost of the first iteration in the inner loop of SAOCIF is $O\left(L \cdot N_{k-1}^{2}\right)$, and $O\left(L \cdot N_{k-1} \cdot \max \left(\delta N_{k}, m\right)\right)$ for the rest of iterations.

\subsection{Compared Methods and Settings}

At first, the original algorithms can only be directly compared if we set $C_{\max }=1$, just because in EM-ELMs the inner while loop of SAOCIF is not carried out. However, in 
order to make a more general comparison with the only difference residing on whether the candidates are randomly generated or taken randomly from the input patterns (i.e. random versus input strategy), we have defined two settings. In the former, $C_{\max }=1$, so the original EM-ELMs are confronted with a very limited version of SV-SFNNs in which a randomly selected input (not the best) yields the single candidate. In the latter, $C_{\max }=59$, so an extended version of EM-ELMs (with the upgrade of selecting the best random candidate among $C_{\max }$ at each step) is confronted with a not so limited version of SV-SFNNs in which not the best of the remaining candidates but the best of a randomly selected subset (of size $C_{\max }$ ) of the remaining candidates is added. The choice of $C_{\max }=59$ is justified because, in order to obtain a candidate that is with probability 0.95 among the best $5 \%$ of all candidates, a random subset of size $\lceil\log 0.05 / \log 0.95\rceil=59$ suffices [14].

These settings allow to make a fair comparison of EM-ELMs and SV-SFNNs, since they work in the same conditions and taking the same computation time.

\subsection{Software}

We have used our own implementation in $\mathrm{C}$ setting the algorithm parameters as explained in the preceding paragraph. The optimal output-layer weights were computed using (10) and (11).

\subsection{Data Sets}

The comparison was performed using 20 benchmark data sets, 10 for classification and 10 for regression problems. The classification data sets were Australian Credit, Splice-junction Gene Sequences, German Credit, Ionosphere, Iris, Landsat Satellite (Satimage), Image Segmentation, Sonar, Vehicle Silhouettes and Wine, and can be found in the UCI repository [1]. The features of these data sets are summarized in Table 1. The regression data sets were Abalone, Auto Price, Boston Housing, California Housing, Census House, Delta Ailerons, Delta Elevators, Machine CPU, Servo and Stock, that can be found at http://www.liaad.up.pt/ ltorgo/ Regression/Datasets.html. The features of these data sets are summarized in Table 2.

\subsection{Methodology}

- Preprocessing. Categorical attributes were converted to dummy variables. The rest of the attributes (including the target variable for regression data sets) were scaled to mean zero and variance one.

- Random weights. In the random strategy, hidden-layer weights were uniformly chosen within the same range of values that the input values (after scaling). In this way, the ranges of the hidden-layer weights were the same for both strategies.

- Activation functions. Three types were used: Gaussian RBF (2), sigmoid additive (3) and sin additive (4) units, but with a further multiplicative positive 


\begin{tabular}{lrrr}
\hline Data Set & \#Inputs & \#Exa. & \#Classes \\
\hline Australian & 43 & 690 & 2 \\
Gene & 120 & 3175 & 3 \\
German & 56 & 1000 & 2 \\
Ionosphere & 34 & 351 & 2 \\
Iris & 4 & 150 & 3 \\
Satimage & 36 & 6435 & 6 \\
Segmentation & 16 & 2310 & 7 \\
Sonar & 60 & 208 & 2 \\
Vehicle & 18 & 846 & 4 \\
Wine & 13 & 178 & 3 \\
\hline
\end{tabular}

Table 1: Features of the classification benchmark data sets

\begin{tabular}{lrrrr}
\hline Data Set & \#Inputs & \#Exa. & Target Mean & StdDev \\
\hline Abalone & 8 & 4177 & 9.93 & 3.22 \\
Auto Price & 15 & 159 & 11445.73 & 5877.85 \\
Boston Housing & 13 & 506 & 22.53 & 9.20 \\
Calif. Housing & 8 & 20640 & 206854.97 & 115395.58 \\
Census House & 8 & 22784 & 50073.10 & 52846.16 \\
Delta Ailerons & 5 & 7129 & -0.000007 & 0.0003 \\
Delta Elevators & 6 & 9517 & -0.000133 & 0.0023 \\
Machine CPU & 6 & 209 & 105.62 & 160.83 \\
Servo & 4 & 167 & 1.39 & 1.56 \\
Stock & 9 & 950 & 46.99 & 6.54 \\
\hline
\end{tabular}

Table 2: Features of the regression benchmark data sets

parameter $\gamma$ introduced for a wider search. Specifically, $\gamma$ multiplies the distance $\left\|\mathbf{x}-\boldsymbol{\omega}_{i}\right\|$ in the RBF units and the scalar product $\boldsymbol{\omega}_{i} \cdot \mathbf{x}$ in the additive units.

- Parameters and model selection. A hidden-unit candidate weight vector was not considered valid if the associated linear equations system could not be solved or if the 1-norm of the solution (the output-layer weights) was greater than a certain value $M$. This can be seen as a form of regularization. $M$ was set to 1024 . We fixed $N_{\max }=99$ and $\epsilon=0$, so that $N_{\max }$ hidden units were always added. In order to get an adequate value for the $\gamma$ parameter, a search was performed ranging $\gamma$ from $2^{-10}$ to $2^{5}$. The same search was performed for all the models, and repeated for every activation function.

- Model training and testing. The methods were trained and tested over 30 training-validation-test different random partitions ( $80 \%$ training, $10 \%$ validation, $10 \%$ test) of the whole data set. For every configuration (defined by a given strategy, $C_{\max }$, activation function and $\gamma$ ), the networks with the lowest errors in the validation subsets were selected as the final models. For classification data 
sets, the accuracies of the final models were given by the average accuracies measured in the test subsets. For regression data sets, the performance of the finel models was measured by the following Normalized Squared Error (NSE) [2]:

$$
N S E=\frac{\sum_{j=1}^{K}\left\|f\left(\mathbf{x}_{j}\right)-\mathbf{t}_{j}\right\|^{2}}{\sum_{j=1}^{K}\left\|\overline{\mathbf{t}}-\mathbf{t}_{j}\right\|^{2}},
$$

where $K$ is the number of examples in the test subset, $f(\mathbf{x})$ is the final model and $\overline{\mathbf{t}}$ is the mean target value in the test subset. The sizes of the final models are defined by their average number of hidden units.

\subsection{Experimental Results}

Tables 3 and 4 show the average accuracies of the best final models (among all $\gamma$ ) for the two strategies (input and random) and the three activation functions tried (Gaussian RBF, sin additive, sigmoid additive) using the methodology previously described for the 10 classification data sets studied. Table 3 displays the results of the methods for $C_{\max }=1$, where the input strategy is fully comparable to EM-ELMs, and Table 4 displays the results for $C_{\max }=59$. For the 10 regression data sets studied, tables 5 and 6 show the average NSE of the best final models for $C_{\max }=1$ and $C_{\max }=59$ respectively. Tables 7, 8, 9 and 10 show the average number of hidden units in the final models selected for each combination of strategy and activation function for $C_{\max }=1$ and $C_{\max }=59$.

It can be observed that Iris and Wine data sets correspond to easy problems that have been learnt perfectly using both strategies. For the other data sets, test results look similar between the two strategies in some cases and a superior performance of the input strategy can be appreciated in the rest, except for the Stock data set. Not surprisingly, the best values for each strategy and data set are included in tables 4 and 6 (i.e. they have been obtained using $C_{\max }=59$ ) except, again, for the Stock data set. Note that the low number of hidden units of the best final models for the Stock data set (see tables 9 and 10) is a clear indication of a strong tendency to overfitting in this data set.

In order to obtain an objective statistical measure, a Student's t-test was applied to each data set to check if the difference between the best mean results of the two strategies was statistically significant ( $\mathrm{p}$-value $=0.05$, i.e. confidence of $95 \%$ ). The test was applied to the best models obtained with $C_{\max }=59$, which are marked in bold (all of them are the best results for each strategy and data set, except for the Stock data set). Results of the test are shown in table 11. In six of the classification data sets (Australian Credit, Gene, Ionosphere, Satimage, Segmentation, Sonar) and six of the regression data sets (Auto Price, California Housing, Census House, Delta Elevators, Machine CPU, Servo) the t-test gave a significant difference with a superior mean accuracy of the input strategy, whereas no significant difference was found in the other ones (German Credit and Vehicle for classification and Abalone, Boston Housing, Delta Ailerons and Stock for regression). Small differences (see Gene, California Housing, Delta Elevators, for example) are sometimes more significant than larger ones (Stock, for example) because the former have very small variances. 
Although no clear trend is observed about the number of hidden units selected by both strategies (it depends quite a lot on the specific activation function), the input strategy seems to need more units than the random strategy in the case of Gaussian RBF hidden units (this can be seen easily in Table 8). Regarding the number of candidates, final models obtained with $C_{\max }=1$ usually have more hidden units than those obtained with $C_{\max }=59$.

As a reference, the mean execution times to obtain every final model were 58.8 seconds for Satimage and 99 seconds for Census House (carried out in a node of a computation cluster with Intel ${ }^{\circledR}$ Xeon ${ }^{\circledR}$ CPUs at $\left.2.66 \mathrm{GHz}\right)$.

\section{Conclusions and Future Work}

First, it has been shown that EM-ELMs can be cast as a particular case of the SAOCIF method (with Random strategy) for constructing SLFNs. The two only differences between the original EM-ELMs proposed in [4] and the SAOCIF method with Random strategy proposed previously in [12] are: $i$ ) the incremental method in which the same optimal (in a least squares sense) output-layer weights are recalculated each time a random hidden unit is added (or tested), and ii) the number of random candidates tested for each hidden unit. Regarding the first difference, it has been demonstrated in Section 3.1 that the cost of the computation described in [4] is greater than the corresponding one in the (generalized) method described in [12]. Since both methods compute the same optimal output-layer weights, there is no problem in using the more efficient one also for EM-ELMs, thus removing the difference. The second difference can also be eliminated either by restricting SAOCIF to test a single candidate at each step (as the original EM-ELMs do) or extending EM-ELMs to test some number of random candidates at each step and select the best of them (as SAOCIF does). Both possibilities have been explored in the experimental study carried out in this work.

Second, we have claimed that an alternative sequential method to construct SLFNs can be based on selecting the hidden-layer weights among the input vectors in the training set. This method, which has been referred to as SV-SFNNs [13] or SAOCIF with Input strategy [12], is essentially equivalent to the Orthogonal Least Squares Learning algorithm [3] and to the Kernel Matching Pursuit with pre-fitting method [16]. In order to assess the relative performance of both approaches (EM-ELMs $v s$. SV-SFNNs) in a fair manner, an empirical study has been realized on twenty benchmark data sets, 10 for classification and 10 for regression, under the same conditions and using the same software.

The experimental comparison between EM-ELMs and SV-SFNNs presented in the paper draws two interesting conclusions that can be further investigated in future research. The first one is that selecting the hidden-layer weights as a subset of the input data, even if this selection is done randomly, yields better generalization results than selecting the hidden-layer weights in a purely random manner from scratch (like EMELMs do). As discussed at the end of Section 1, this is not an obvious result. Indeed, no statistically significant difference between the average performances obtained by the two strategies was found in eight of the benchmark problems, but SV-SFNNs showed a statistically significant improvement in generalization performance in the other twelve. 
One might ask whether there is any noticeable difference between these two groups of problems. For classification problems, data sets with a higher number of variables (see Table 1 and imagine for instance an arbitrary threshold of 20 inputs) were the ones in which SV-SFNNs outperformed EM-ELMs (with the exceptions of the German credit and the Segmentation problems). Although this can be considered as a reasonable result, which may be justified by the difficulty in finding adequate decision boundaries in high-dimensional input spaces from randomly distributed hidden-layer weights, the underlying hypothesis needs further validation in future studies. For regression problems this trend is not so clear.

The second conclusion of the experimental study is that, independently of the strategy used (input or random), the number of candidates for the hidden-layer weights is a parameter that controls the trade-off between the generalization performance, the computational cost and the number of hidden units of the final models. In general terms, by increasing the number of candidates at each step of the sequential algorithm (recall that in the originally proposed EM-ELMs this number is 1), the generalization is improved and the final number of hidden units is reduced, at the expense of a higher training time. However, as pointed in Section 3.1, the computational cost of trying $C$ candidates is lower than $C$ times the cost of trying a single candidate, due to the incremental way in which the optimal output-layer weights are calculated.

\section{References}

[1] A. Asuncion and D. J. Newman. UCI machine learning repository, 2007. University of California, Irvine, School of Information and Computer Science. http://www.ics.uci.edu/ mlearn/MLRepository.html.

[2] C. M. Bishop. Neural Networks for Pattern Recognition. Oxford University Press Inc., New York, 1995.

[3] S. Chen, C. F. N. Cowan, and P. M. Grant. Orthogonal Least Squares Learning Algorithm for Radial Basis Function Networks. IEEE Transactions on Neural Networks, 2(2):302-309, 1991.

[4] G. Feng, G. B. Huang, Q. Lin, and R. Gay. Error Minimized Extreme Learning Machine with Growth of Hidden Nodes and Incremental Learning. IEEE Transactions on Neural Networks, 20(8):1352-1357, 2009.

[5] G. B. Huang, L. Chen, and C. K. Siew. Universal Approximation using Incremental Constructive Feedforward Networks with Random Hidden Nodes. IEEE Transactions on Neural Networks, 17(4):879-892, 2006.

[6] G. B. Huang, Q. Y. Zhu, and C. K. Siew. Extreme Learning Machine: Theory and Applications. Neurocomputing, 70(1-3):489-501, 2006.

[7] S. S. Keerthi, O. Chapelle, and D. DeCoste. Building Support Vector Machines with Reduced Classifier Complexity. Journal of Machine Learning Research, 7:1493-1515, 2006. 
[8] T. Y. Kwok and D. Y. Yeung. Constructive Algorithms for Structure Learning in Feedforward Neural Networks for Regression Problems. IEEE Transactions on Neural Networks, 8(3):630-645, 1997.

[9] Y. Miche, A. Sorjamaa, P. Bas, O. Simula, C. Jutten, and A. Lendasse. OP-ELM: Optimally Pruned Extreme Learning Machine. IEEE Transactions on Neural Networks, 21(1):158-162, 2010.

[10] J. Platt. A Resource-Allocating Network for Function Interpolation. Neural Computation, 3(2):213-225, 1991.

[11] E. Romero and R. Alquézar. A New Incremental Method for Function Approximation using Feed-forward Neural Networks. In International Joint Conference on Neural Networks, volume 2, pages 1968-1973, 2002.

[12] E. Romero and R. Alquézar. A Sequential Algorithm for Feed-forward Neural Networks with Optimal Coefficients and Interacting Fr equencies. Neurocomputing, 69(13-15):1540-1552, 2006.

[13] E. Romero and D. Toppo. Comparing Support Vector Machines and Feed-forward Neural Networks with Similar Hidden-layer Weights . IEEE Transactions on Neural Networks, 18(3):959-963, 2007.

[14] A. J. Smola and B. Schölkopf. Sparse Greedy Matrix Approximation for Machine Learning. In International Conference on Machine Learning, pages 911-918, 2000.

[15] V. N. Vapnik. The Nature of Statistical Learning Theory. Springer-Verlag, NY, 1995.

[16] P. Vincent and Y. Bengio. Kernel Matching Pursuit. Machine Learning, 48(13):165-187, 2002. Special Issue on New Methods for Model Combination and Model Selection.

[17] Liang N. Y., G. B. Huang, P. Saratchandran, and N. Sundararajan. A Fast and Accurate Online Sequential Learning Algorithm for Feedforward Networks. IEEE Transactions on Neural Networks, 17(6):1411-1423, 2006.

[18] L. Yingwei, N. Sundararajan, and P. Saratchandran. A Sequential Learning Scheme for Function Approximation using Minimal Radial Basis Function Neural N etworks. Neural Computation, 9(2):461-478, 1997. 


\begin{tabular}{lcccccc}
\hline \multirow{2}{*}{ Data Set } & \multicolumn{2}{c}{ Gaussian RBF } & \multicolumn{2}{c}{ Sin MLP } & \multicolumn{2}{c}{ Sigmoid MLP } \\
\cline { 2 - 7 } & Input & Rand. & Input & Rand. & Input & Rand. \\
\hline Australian & 83.86 & 83.00 & 83.67 & 83.14 & 84.25 & 83.48 \\
Gene & 84.71 & 83.38 & 84.75 & 83.13 & 84.82 & 82.95 \\
German & 77.37 & 78.23 & 77.73 & 77.20 & 77.83 & 77.10 \\
Ionosphere & 93.87 & 90.19 & 90.10 & 88.67 & 90.19 & 88.67 \\
Iris & 100 & 99.11 & 100 & 99.78 & 100 & 99.56 \\
Satimage & 82.98 & 80.57 & 79.17 & 77.73 & 79.40 & 77.62 \\
Segmentation & 86.62 & 81.65 & 86.42 & 86.08 & 86.70 & 86.39 \\
Sonar & 89.84 & 80.83 & 77.17 & 76.17 & 77.50 & 75.83 \\
Vehicle & 85.48 & 85.52 & 86.11 & 84.80 & 85.87 & 85.20 \\
Wine & 99.61 & 100 & 99.80 & 100 & 100 & 100 \\
\hline \multicolumn{7}{r}{} \\
\end{tabular}

Table 3: Comparison of average test accuracy for classification data sets - One candidate

\begin{tabular}{lcccccc}
\hline & \multicolumn{2}{c}{ Gaussian RBF } & \multicolumn{2}{c}{ Sin MLP } & \multicolumn{2}{c}{ Sigmoid MLP } \\
\cline { 2 - 7 } Data Set & Input & Rand. & Input & Rand. & Input & Rand. \\
\hline Australian & 84.49 & 83.48 & 83.77 & 83.43 & $\mathbf{8 5 . 0 2}$ & $\mathbf{8 3 . 8 2}$ \\
Gene & $\mathbf{8 6 . 6 0}$ & $\mathbf{8 6 . 0 5}$ & 86.34 & 85.86 & 86.36 & 85.92 \\
German & 77.13 & $\mathbf{7 8 . 3 3}$ & 77.60 & 77.30 & $\mathbf{7 8 . 0 3}$ & 77.37 \\
Ionosphere & $\mathbf{9 3 . 9 0}$ & $\mathbf{9 0 . 6 7}$ & 89.52 & 88.67 & 90.00 & 88.83 \\
Iris & 100 & 100 & 100 & 100 & 100 & 100 \\
Satimage & $\mathbf{8 6 . 3 5}$ & $\mathbf{8 3 . 3 1}$ & 82.83 & 77.56 & 81.73 & 77.50 \\
Segmentation & $\mathbf{8 8 . 5 6}$ & 83.39 & 86.61 & 86.64 & 87.30 & $\mathbf{8 6 . 8 3}$ \\
Sonar & $\mathbf{9 6 . 5 0}$ & $\mathbf{8 1 . 8 3}$ & 87.67 & 76.67 & 75.00 & 74.17 \\
Vehicle & 86.67 & 85.56 & $\mathbf{8 6 . 8 7}$ & 86.11 & 86.75 & $\mathbf{8 6 . 6 3}$ \\
Wine & 100 & 100 & 100 & 100 & 100 & 100 \\
\hline
\end{tabular}

Table 4: Comparison of average test accuracy for classification data sets - Best of 59 candidates

\begin{tabular}{lllllll}
\hline & \multicolumn{2}{c}{ Gaussian RBF } & \multicolumn{2}{c}{ Sin MLP } & \multicolumn{2}{c}{ Sigmoid MLP } \\
\cline { 2 - 7 } Data Set & Input & Rand. & Input & Rand. & Input & Rand. \\
\hline Abalone & 0.517 & 0.526 & 0.521 & 0.553 & 0.514 & 0.550 \\
Auto Price & 0.341 & 1.570 & 0.270 & 0.870 & 0.280 & 0.917 \\
Boston Housing & 0.725 & 0.726 & 0.941 & 1.166 & 0.871 & 1.162 \\
Calif. Housing & 0.344 & 0.369 & 0.367 & 0.367 & 0.367 & 0.366 \\
Census House & 0.404 & 1.070 & 0.484 & 0.580 & 0.457 & 0.502 \\
Delta Ailerons & 0.292 & 0.297 & 0.305 & 0.301 & 0.304 & 0.302 \\
Delta Elevators & 0.376 & 0.377 & 0.375 & 0.377 & 0.376 & 0.376 \\
Machine CPU & 0.364 & 0.404 & 0.358 & 0.394 & 0.330 & 0.378 \\
Servo & 0.214 & 0.248 & 0.228 & 0.329 & 0.246 & 0.337 \\
Stock & 1.689 & 1.689 & 2.023 & 9.883 & 8.897 & 7.796 \\
\hline
\end{tabular}

Table 5: Comparison of average test NSE for regression data sets - One candidate

\begin{tabular}{lllllll}
\hline & \multicolumn{2}{c}{ Gaussian RBF } & \multicolumn{2}{c}{ Sin MLP } & \multicolumn{2}{c}{ Sigmoid MLP } \\
\cline { 2 - 7 } Data Set & Input & Rand. & Input & Rand. & Input & Rand. \\
\hline Abalone & 0.512 & $\mathbf{0 . 5 2 4}$ & 0.512 & 0.537 & $\mathbf{0 . 5 1 1}$ & 0.537 \\
Auto Price & $\mathbf{0 . 1 7 7}$ & 0.840 & 0.275 & $\mathbf{0 . 4 5 6}$ & 0.299 & 0.517 \\
Boston Housing & $\mathbf{0 . 6 5 7}$ & $\mathbf{0 . 6 2 0}$ & 0.762 & 0.898 & 0.745 & 0.921 \\
Calif. Housing & $\mathbf{0 . 3 3 2}$ & $\mathbf{0 . 3 4 5}$ & 0.368 & 0.366 & 0.369 & 0.365 \\
Census House & $\mathbf{0 . 3 8 3}$ & 0.508 & 0.425 & 0.528 & 0.403 & $\mathbf{0 . 4 7 0}$ \\
Delta Ailerons & $\mathbf{0 . 2 9 3}$ & $\mathbf{0 . 2 9 5}$ & 0.303 & 0.303 & 0.299 & 0.301 \\
Delta Elevators & 0.375 & 0.376 & $\mathbf{0 . 3 7 4}$ & $\mathbf{0 . 3 7 5}$ & 0.375 & 0.375 \\
Machine CPU & $\mathbf{0 . 2 9 9}$ & $\mathbf{0 . 3 4 8}$ & 0.370 & 0.385 & 0.345 & 0.380 \\
Servo & $\mathbf{0 . 1 5 8}$ & $\mathbf{0 . 2 0 6}$ & 0.185 & 0.289 & 0.171 & 0.300 \\
Stock & $\mathbf{1 . 7 1 4}$ & $\mathbf{1 . 6 0 8}$ & 2.896 & 2.147 & 3.318 & 1.807 \\
\hline
\end{tabular}

Table 6: Comparison of average test NSE for regression data sets - Best of 59 candidates 


\begin{tabular}{lcrrrrr}
\hline \multirow{2}{*}{ Data Set } & \multicolumn{2}{c}{ Gaussian RBF } & \multicolumn{2}{c}{ Sin MLP } & \multicolumn{2}{c}{ Sigmoid MLP } \\
\cline { 2 - 7 } & Input & Rand. & Input & Rand. & Input & Rand. \\
\hline Australian & 43.53 & 30.33 & 25.17 & 37.40 & 61.33 & 44.90 \\
Gene & 97.07 & 98.87 & 97.40 & 98.60 & 97.20 & 98.00 \\
German & 40.47 & 43.23 & 44.53 & 33.87 & 36.77 & 36.80 \\
Ionosphere & 73.10 & 40.63 & 19.40 & 24.30 & 14.97 & 24.60 \\
Iris & 55.97 & 31.63 & 3.87 & 3.60 & 3.87 & 3.67 \\
Satimage & 95.30 & 96.80 & 97.93 & 97.13 & 95.47 & 97.73 \\
Segmentation & 41.53 & 25.90 & 63.10 & 74.30 & 53.40 & 82.07 \\
Sonar & 66.53 & 64.00 & 47.67 & 43.97 & 38.13 & 42.10 \\
Vehicle & 74.77 & 38.00 & 67.30 & 61.73 & 64.03 & 63.97 \\
Wine & 11.57 & 11.77 & 7.73 & 10.00 & 7.90 & 10.90 \\
\hline
\end{tabular}

Table 7: Average number of hidden units for classification data sets - One candidate

\begin{tabular}{lrrrrrr}
\hline & \multicolumn{2}{c}{ Gaussian RBF } & \multicolumn{2}{c}{ Sin MLP } & \multicolumn{2}{c}{ Sigmoid MLP } \\
\cline { 2 - 7 } Data Set & Input & Rand. & Input & Rand. & Input & Rand. \\
\hline Australian & 50.63 & 23.33 & 15.53 & 32.67 & 24.93 & 21.77 \\
Gene & 95.23 & 89.87 & 90.33 & 87.07 & 88.33 & 84.80 \\
German & 12.20 & 12.20 & 12.97 & 14.23 & 13.83 & 16.90 \\
Ionosphere & 65.73 & 29.03 & 10.40 & 12.10 & 12.53 & 12.67 \\
Iris & 19.13 & 4.50 & 3.87 & 2.97 & 3.97 & 2.77 \\
Satimage & 97.67 & 89.73 & 96.23 & 96.83 & 95.40 & 97.43 \\
Segmentation & 95.83 & 32.00 & 75.33 & 63.57 & 91.87 & 68.27 \\
Sonar & 88.27 & 55.43 & 31.17 & 10.57 & 25.13 & 21.13 \\
Vehicle & 81.73 & 37.13 & 44.80 & 50.77 & 58.37 & 54.27 \\
Wine & 4.90 & 7.13 & 5.00 & 6.13 & 5.13 & 6.23 \\
\hline
\end{tabular}

Table 8: Average number of hidden units for classification data sets - Best of 59 candidates

\begin{tabular}{lrrrrrr}
\hline & \multicolumn{2}{c}{ Gaussian RBF } & \multicolumn{2}{c}{ Sin MLP } & \multicolumn{2}{c}{ Sigmoid MLP } \\
\cline { 2 - 7 } Data Set & Input & Rand. & Input & Rand. & Input & Rand. \\
\hline Abalone & 55.60 & 22.27 & 61.13 & 10.17 & 78.23 & 78.70 \\
Auto Price & 8.40 & 5.07 & 7.43 & 10.70 & 7.53 & 10.80 \\
Boston Housing & 18.57 & 23.23 & 4.03 & 5.00 & 3.80 & 4.50 \\
Calif. Housing & 36.40 & 34.13 & 8.10 & 30.17 & 8.67 & 31.43 \\
Census House & 87.67 & 6.00 & 94.70 & 82.80 & 93.03 & 92.20 \\
Delta Ailerons & 81.40 & 51.63 & 32.03 & 39.70 & 33.67 & 36.33 \\
Delta Elevators & 66.10 & 80.33 & 52.10 & 50.37 & 58.00 & 64.13 \\
Machine CPU & 4.33 & 5.73 & 7.63 & 4.97 & 7.50 & 5.17 \\
Servo & 60.43 & 42.27 & 65.67 & 14.40 & 65.50 & 14.57 \\
Stock & 1.07 & 1.07 & 1.80 & 4.53 & 3.20 & 2.97 \\
\hline
\end{tabular}

Table 9: Average number of hidden units for regression data sets - One candidate

\begin{tabular}{lrrrrrr}
\hline & \multicolumn{2}{c}{ Gaussian RBF } & \multicolumn{2}{c}{ Sin MLP } & \multicolumn{2}{c}{ Sigmoid MLP } \\
\cline { 2 - 7 } Data Set & Input & Rand. & Input & Rand. & Input & Rand. \\
\hline Abalone & 34.60 & 48.17 & 54.67 & 40.13 & 31.70 & 56.23 \\
Auto Price & 7.37 & 4.20 & 3.47 & 5.43 & 3.00 & 4.87 \\
Boston Housing & 11.93 & 14.90 & 1.53 & 2.33 & 1.30 & 8.80 \\
Calif. Housing & 88.87 & 54.00 & 7.57 & 19.07 & 7.10 & 14.47 \\
Census House & 95.13 & 37.00 & 96.43 & 36.97 & 91.23 & 78.27 \\
Delta Ailerons & 54.73 & 34.43 & 21.83 & 30.07 & 72.20 & 72.20 \\
Delta Elevators & 40.17 & 57.90 & 31.50 & 28.97 & 36.20 & 29.20 \\
Machine CPU & 5.37 & 3.97 & 5.97 & 3.07 & 5.27 & 3.53 \\
Servo & 61.57 & 21.17 & 54.00 & 32.47 & 57.37 & 30.63 \\
Stock & 4.93 & 1.00 & 1.10 & 2.07 & 1.13 & 1.33 \\
\hline
\end{tabular}

Table 10: Average number of hidden units for regression data sets - Best of 59 candidates 


\begin{tabular}{lccccc}
\hline & \multicolumn{2}{c}{ Input } & \multicolumn{2}{c}{ Random } & t-test \\
\cline { 2 - 6 } Data Set & Mean & Std & Mean & Std & p-value \\
\hline Australian & 85.02 & 1.6304 & 83.82 & 1.3237 & $\mathbf{0 . 0 0 2 6 1 6}$ \\
Gene & 86.60 & 0.9905 & 86.05 & 0.8409 & $\mathbf{0 . 0 2 2 2 4 6}$ \\
German & 78.03 & 1.9205 & 78.33 & 2.0398 & 0.559828 \\
Ionosphere & 93.90 & 1.9466 & 90.67 & 2.7003 & $\mathbf{0 . 0 0 0 0 0 2}$ \\
Iris & 100 & 0.0 & 100 & 0.0 & - \\
Satimage & 86.35 & 0.6592 & 83.31 & 1.2996 & $\mathbf{0 . 0 0 0 0 0 0}$ \\
Segmentation & 88.56 & 0.9289 & 86.83 & 0.8342 & $\mathbf{0 . 0 0 0 0 0 0}$ \\
Sonar & 96.50 & 8.6253 & 81.83 & 9.6921 & $\mathbf{0 . 0 0 0 0 0 0}$ \\
Vehicle & 86.87 & 2.3103 & 86.63 & 3.2014 & 0.744651 \\
Wine & 100 & 0.0 & 100 & 0.0 & - \\
\hline Abalone & 0.511 & 0.0118 & 0.524 & 0.0348 & 0.053008 \\
Auto Price & 0.177 & 0.0619 & 0.456 & 0.3090 & $\mathbf{0 . 0 0 0 0 3 2}$ \\
Boston Housing & 0.657 & 0.1101 & 0.620 & 0.0954 & 0.172238 \\
Calif. Housing & 0.332 & 0.0092 & 0.345 & 0.0182 & $\mathbf{0 . 0 0 0 8 9 4}$ \\
Census House & 0.383 & 0.0045 & 0.470 & 0.0323 & $\mathbf{0 . 0 0 0 0 0 0}$ \\
Delta Ailerons & 0.293 & 0.0057 & 0.295 & 0.0031 & 0.057887 \\
Delta Elevators & 0.374 & 0.0020 & 0.375 & 0.0020 & $\mathbf{0 . 0 2 3 5 9 4}$ \\
Machine CPU & 0.299 & 0.0431 & 0.348 & 0.0981 & $\mathbf{0 . 0 1 5 5 4 7}$ \\
Servo & 0.158 & 0.0308 & 0.206 & 0.0538 & $\mathbf{0 . 0 0 0 1 1 6}$ \\
Stock & 1.714 & 0.0004 & 1.608 & 0.5498 & 0.298702 \\
\hline & & & & &
\end{tabular}

Table 11: Results of Student's t-test for all data sets - Best of 59 candidates (and best activation function) 